\title{
Reducible Curves on Rational Surfaces
}

\author{
Hideo KOJIMA and Takeshi TAKAHASHI
}

Niigata University and Nagaoka National College of Technology

(Communicated by S. Nakajima)

\section{Introduction}

Let $k$ be an algebraically closed field of characteristic zero. Throughout the present article we fix $k$ as the ground field. Let $X$ be a smooth projective surface and $B$ a reduced curve on $X$. Then we define the $m$-genus $P_{m}[B]$ and the Kodaira dimension $\kappa[B]$ as follows (cf. [4]): Let $f: V \rightarrow X$ be a birational morphism such that the strict transform $D=$ $f^{\prime}(B)$ becomes a disjoint union of smooth curves. Then $P_{m}[B]:=h^{0}\left(V, m\left(D+K_{V}\right)\right)$ and $\kappa[B]:=\kappa\left(D+K_{V}, V\right)$, where $K_{V}$ is the canonical divisor on $V$ and $\kappa\left(D+K_{V}, V\right)$ is the $\left(D+K_{V}\right)$-dimension of $V$ (cf. [1]). Note that $P_{m}[B]=\bar{P}_{m}(V-D)$ and $\kappa[B]=\bar{\kappa}(V-D)$, where $\bar{P}_{m}(V-D)$ (resp. $\left.\bar{\kappa}(V-D)\right)$ denotes the logarithmic $m$-genus (resp. the logarithmic Kodaira dimension) of $V-D$ (cf. [1] and [11]).

Pairs $(X, B)$ of smooth projective rational surfaces $X$ and irreducible curves $B$ on $X$ were studied from the viewpoint of birational geometry by litaka [2, 5], Matsuda [9] and the others. In [3] and [4], Iitaka studied reducible curves $B$ on smooth projective rational surfaces such that $\#(B)=2$, where $\#(B)$ is the number of irreducible components of $B$. In particular, he proved the following result.

THEOREM 1.1. ([4]) Let $B$ be a reduced curve on a smooth projective rational surface $X$ with $\#(B) \leq 2$. Then $\kappa[B]=-\infty$ if and only if $P_{2}[B]=0$.

In the present article, by using the theory of open algebraic surfaces, we study reduced curves on smooth projective rational surfaces. In $\S 3$, we give a simple proof of Theorem 1.1 by using the structure theorems of open algebraic surfaces (cf. §2). In §4, we study the case where $B$ consists of two rational curves and $\kappa[B]=0$ or 1 and give a structure theorem of such a pair, which improves [4, Proposition 3]. In $\S 5$, we consider the relation of $P_{m}[B]$ and $\kappa[B]$ when $\#(B) \leq 4$.

The authors would like to thank the referee for giving useful comments.

Received March 3, 2005; revised August 29, 2005

Mathematics Subject Classification (2000): Primary 14J26; Secondary 14E05. 


\section{Preliminary results}

We recall some basic notions in the theory of peeling. For more details, see [12, Chapter 2]. Let $(X, B)$ be a pair of a smooth projective surface $X$ and an SNC-divisor $B$ (i.e., $B$ is a reduced divisor on $X$ and has only simple normal crossings as singularities). We call such a pair $(X, B)$ an $S N C$-pair. A connected curve $T$ consisting of irreducible components of $B$ (a connected curve in $B$, for short) is a twig if the dual graph of $T$ is a linear chain and $T$ meets $B-T$ in a single point at one of the end components of $T$, the other end of $T$ is called the tip of $T$. A connected curve $R$ (resp. $F$ ) in $B$ is a $\operatorname{rod}$ (resp. fork) if $R$ (resp. $F$ ) is a connected component of $B$ and the dual graph of $R$ (resp. $F$ ) is a linear chain (resp. the dual graph of the exceptional curves of the minimal resolution of a non-cyclic quotient singularity). A connected curve $E$ in $B$ is rational (resp. admissible) if each irreducible component of $E$ is rational (resp. if there are no $(-1)$-curves in Supp $(E)$ and the intersection matrix of $E$ is negative definite). An admissible rational twig $T$ in $B$ is maximal if $T$ is not extended to an admissible rational twig with more irreducible components of $B$.

Let $\left\{T_{\lambda}\right\}$ (resp. $\left\{R_{\mu}\right\},\left\{F_{\nu}\right\}$ ) be the set of all admissible rational maximal twigs (resp. all admissible rational rods, all admissible rational forks), where no irreducible components of $T_{\lambda}$ 's belong to $R_{\mu}$ 's or $F_{v}$ 's. Then there exists a unique decomposition of $B$ as a sum of effective $\mathbf{Q}$-divisors $B=B^{\#}+\mathrm{Bk}(B)$ such that the following two conditions i) and ii) are satisfied:

i) $\operatorname{Supp}(\mathrm{Bk}(B))=\left(\cup_{\lambda} T_{\lambda}\right) \cup\left(\cup_{\mu} R_{\mu}\right) \cup\left(\cup_{\nu} F_{\nu}\right)$.

ii) $\left(B^{\#}+K_{X} \cdot Z\right)=0$ for every irreducible component $Z$ of Supp $(\operatorname{Bk}(B))$.

We call the Q-divisor Bk $(B)$ the bark of $B$ and say that $B^{\#}+K_{X}$ is produced by the peeling of $B$.

Definition 2.1. Let $(X, B)$ be an SNC-pair. Then $(X, B)$ is said to be almost minimal if, for every irreducible curve $C$ on $X$, either $\left(B^{\#}+K_{X} \cdot C\right) \geq 0$ or $\left(B^{\#}+K_{X} \cdot C\right)<0$ and the intersection matrix of $C+\mathrm{Bk}(B)$ is not negative definite.

LEMMA 2.2. Let $(X, B)$ be an SNC-pair. Then there exists a birational morphism $\mu: X \rightarrow W$ onto a smooth projective surface $W$ such that the following four conditions (i) (iv) are satisfied:

(i) $C:=\mu_{*}(B)$ is an SNC-divisor.

(ii) $\mu_{*}(\mathrm{Bk}(B)) \leq \mathrm{Bk}(C)$ and $\mu_{*}\left(B^{\#}+K_{X}\right) \geq C^{\#}+K_{W}$.

(iii) $\quad \bar{P}_{n}(X-B)=\bar{P}_{n}(W-C)$ for every integer $n \geq 1$. In particular, $\bar{\kappa}(X-B)=$ $\bar{\kappa}(W-C)$.

(iv) The pair $(W, C)$ is almost minimal.

Proof. See [12, Theorem 2.3.11.1 (p. 107)].

In Lemma 2.2, we call the pair $(W, C)$ an almost minimal model of $(X, B)$.

DEFINITION 2.3. Let $(X, B)$ be a pair of a smooth projective surface $X$ and a reduced curve $B$ on $X$. Let $f: W \rightarrow X$ be a birational morphism such that the strict transform $f^{\prime}(B)$ 
becomes a disjoint union of smooth curves and let $(V, D)$ be an almost minimal model of the SNC-pair $\left(W, f^{\prime}(B)\right)$. Then we call the SNC-pair $(V, D)$ an almost minimal model of $(X, B)$.

In order to prove the results in $\S \S 3 \sim 5$, we use the following lemmas frequently (Lemmas $2.4 \sim 2.11$ ).

LEMmA 2.4. Let $(V, D)$ be an almost minimal SNC-pair. Then $\bar{\kappa}(V-D) \geq 0$ if and only if $D^{\#}+K_{V}$ is nef. Moreover, $\left(D^{\#}+K_{V}\right)^{2}>0$ if $\bar{\kappa}(V-D)=2$.

PROOF. See [12, Theorem 3.15.1 (p. 116)].

LEMMA 2.5. Let $(V, D)$ be an SNC-pair. Let $f: V \rightarrow \bar{V}$ be the contraction of Supp (Bk $(D)), \bar{V}$ being a normal projective surface with only quotient singularities, and let $\bar{D}=f_{*}\left(D^{\#}\right)$. Assume that $(V, D)$ is almost minimal and $\bar{\kappa}(V-D)=-\infty$. Then we have either (1) or (2) below:

(1) $V-D$ is affine ruled, i.e., $V-D$ contains a non-empty Zariski open subset isomorphic to $\mathbf{A}^{1} \times U_{0}$, where $U_{0}$ is a smooth curve.

(2) $\rho(\bar{V})=1$, where $\rho(\bar{V})$ denotes the Picard number of $\bar{V}$, and $-\left(\bar{D}+K_{\bar{V}}\right)$ is an ample $\mathbf{Q}$-Cartier divisor.

Proof. See [12, Theorem 3.15.1 (p. 116)].

Lemma 2.6. Let $(V, D)$ be an almost minimal $S N C$-pair with $\bar{\kappa}(V-D)=0$. Then $D^{\#}+K_{V} \equiv 0$.

PROOF. See [12, Corollary 6.1.4 (p. 175)].

LEMMA 2.7. Let $(V, D)$ be an almost minimal $S N C$-pair. Assume that $V$ is a rational surface, $\bar{\kappa}(V-D)=1$ and $\operatorname{Supp}(\operatorname{Bk}(D))=\operatorname{Supp}(D)$. Then a complete linear system $\left|j\left(D^{\#}+K_{V}\right)\right|$ gives rise to an irreducible pencil of elliptic curves $h: V \rightarrow \mathbf{P}^{1}$ for a sufficiently large integer $j$, by taking, if necessary, the Stein factorization of $\Phi_{\left|j\left(D^{\#}+K_{V}\right)\right|}$. Moreover, every component of $D$ is contained in a fiber of $h$.

Proof. By [12, Theorem 6.1.5 (p. 175)], $\left|j\left(D^{\#}+K_{V}\right)\right|$ defines an irreducible pencil of elliptic curves or rational curves $h: V \rightarrow \mathbf{P}^{1}$ for a sufficiently large integer $j$ by taking, if necessary, the Stein factorization of $\Phi_{\left|j\left(D^{\#}+K_{V}\right)\right|}$. If $h$ is a $\mathbf{P}^{1}$-fibration, then $D$ contains a component $D_{1}$ which is a section or a 2 -section of $h$. Since $\left(D^{\#}+K_{V} \cdot F\right)=0$ for a general fiber $F$ of $h$, the coefficient of $D_{1}$ in $D^{\#}$ must be one, which contradicts the hypothesis $\operatorname{Supp}(\operatorname{Bk}(D))=\operatorname{Supp}(D)$. Hence $h$ is an elliptic fibration. Since $\left(D^{\#}+K_{V} \cdot D\right)=0$ and $D^{\#}+K_{V}$ is nef by Lemma 2.4, every component of $D$ is contained in a fiber of $h$.

Lemma 2.8. Let $(V, D)$ be an SNC-pair. Assume that $V$ is a rational surface. Then $\bar{p}_{g}(V-D)\left(:=\bar{P}_{1}(V-D)\right)=0$ if and only if every irreducible component of $D$ is a rational curve and the dual graph of each connected component of $D$ is a tree. 
ProOF. See [11, Lemma I.2.1.3].

LEMMA 2.9. Let $(V, D)$ be an SNC-pair. Assume that $V$ is a rational surface and each connected component $D^{\prime}$ of $D$ is a (-2)-rod or a (-2)-fork (i.e., $D^{\prime}$ is an admissible rational rod or fork consisting only of $(-2)$-curves). Then $\bar{\kappa}(V-D)=-\infty$.

Proof. By the assumption, $D^{\#}=0$. Then, for any integer $m>0$, we have $\bar{P}_{m}(V-$ $D)=h^{0}\left(V, m\left(D+K_{V}\right)\right)=h^{0}\left(V, m\left(D^{\#}+K_{V}\right)\right)=h^{0}\left(V, m K_{V}\right)=0$. Hence, $\bar{\kappa}(V-D)=$ $-\infty$.

LEMMA 2.10. Let $(V, D)$ be an almost minimal $S N C$-pair with $\bar{\kappa}(V-D) \geq 0$. Then all the maximal rational twigs and rational rods of $D$ are admissible.

Proof. Suppose that $D$ has either a non-admissible maximal rational twig or a nonadmissible rational rod. Then $D$ has a rational curve $D_{1}$ such that the following three conditions are satisfied:

(i) $\left(D_{1}^{2}\right) \geq-1$.

(ii) $\left(D_{1} \cdot D-D_{1}\right) \leq 2$.

(iii) If $\left(D_{1} \cdot D-D_{1}\right)=2$, then $D$ has an admissible rational twig $T$ with $\left(T \cdot D_{1}\right)=1$.

Since $(V, D)$ is almost minimal, we may assume that $\left(D_{1}^{2}\right) \geq 0$. It then follows from $[12$, Corollary 2.11 .1 (p. 82)] that $\bar{\kappa}(V-D)=-\infty$, a contradiction.

LEMMA 2.11. Let $(V, D)$ be an almost minimal $S N C$-pair. Assume that $V$ is a rational surface and $D$ is an admissible rational rod or twig. Then $\bar{\kappa}(V-D) \geq 0$ if and only if $\bar{P}_{2}(V-D)>0$.

Proof. It suffices to show the "only if" part. Let $D=\sum_{i} D_{i}$ be the irreducible decomposition of $D$ and put $D^{\#}=\sum_{i} d_{i} D_{i}$, where $d_{i} \in \mathbf{Q}$. By Lemma 2.9, $D$ is neither a (-2)-rod nor a (-2)-fork. So, $0<d_{i}<1$ for any $i$ (cf. [12, Chapter 2, §3]).

Put $\beta_{i}:=-\left(D_{i}^{2}\right)$. Since $(V, D)$ is almost minimal, Lemma 2.4 implies that

$$
0 \leq\left(D^{\#}+K_{V}\right)^{2}=\left(K_{V} \cdot D^{\#}+K_{V}\right)=\left(K_{V}^{2}\right)+\sum_{i} d_{i}\left(\beta_{i}-2\right)
$$

Since $0<d_{i}<1$ for any $i$, Supp $(D)$ contains a curve with self-intersection number $\leq-3$ and $V$ is a rational surface, we have

$$
\begin{aligned}
h^{0}\left(V, D+2 K_{V}\right)+h^{2}\left(V, D+2 K_{V}\right) & \geq \frac{1}{2}\left(D+2 K_{V} \cdot D+K_{V}\right)+1 \\
& =\left(K_{V} \cdot D+K_{V}\right) \\
& =\left(K_{V}^{2}\right)+\sum_{i}\left(\beta_{i}-2\right) \\
& >\left(K_{V}^{2}\right)+\sum_{i} d_{i}\left(\beta_{i}-2\right) \geq 0 .
\end{aligned}
$$


Here we note that $h^{2}\left(V, D+2 K_{V}\right)=h^{0}\left(V,-\left(D+K_{V}\right)\right)=0$. Indeed, if $h^{0}(V,-(D+$ $\left.\left.K_{V}\right)\right)>0$, then $D+K_{V} \sim 0$ because $\bar{\kappa}(V-D) \geq 0$. So $\bar{p}_{g}(V-D)=1$, which is a contradiction. Therefore, $\bar{P}_{2}(V-D)\left(\geq h^{0}\left(V, D+2 K_{V}\right)\right)>0$.

\section{A proof of Theorem 1.1}

In this section, we give a simple proof of Theorem 1.1 by using the results in $\S 2$.

Let $B$ be a reduced curve on a smooth projective rational surface $X$. Put $n:=\#(B)(\leq 2)$. Let $(V, D)$ be an almost minimal model of the pair $(X, B)$ (cf. Definition 2.3). Note that $\#(D) \leq n(\leq 2)$. It suffices to prove that $P_{2}[B]>0$ provided $\kappa[B] \geq 0$. Assume that $\kappa[B] \geq 0$. Since $\bar{\kappa}(V-D)=\kappa[B] \geq 0$ and $V$ is a rational surface, we have $D \neq 0$. We may assume that $\bar{p}_{g}(V-D)=0$. Then Lemma 2.8 implies that each component of $D$ is a smooth rational curve and the dual graph of each connected component of $D$ is a tree.

We consider the following two cases separately.

CASE 1: $\#(D)=1$. By Lemma $2.10,\left(D^{2}\right) \leq-2$, i.e., $D$ is an admissible rational rod. So, we have $P_{2}[B]=\bar{P}_{2}(V-D)>0$ by Lemma 2.11 .

CASE 2: $\#(D)=2$. Let $D=D_{1}+D_{2}$ be the irreducible decomposition of $D$ and put $\beta_{i}=-\left(D_{i}^{2}\right)$ for $i=1,2$. If $D$ is connected, then $\left(D_{1} \cdot D_{2}\right)=1$ by the assumption $\bar{p}_{g}(V-D)=0$. Lemma 2.10 then implies that $D$ is an admissible rational rod. Hence, $P_{2}[B]=\bar{P}_{2}(V-D)>0$ by Lemma 2.11 .

Assume that $\left(D_{1} \cdot D_{2}\right)=0$. Then $\beta_{i} \geq 2$ for $i=1,2$ by Lemma 2.10 . So, $D^{\#}=$ $\left(\left(\beta_{1}-2\right) / \beta_{1}\right) D_{1}+\left(\left(\beta_{2}-2\right) / \beta_{2}\right) D_{2}$. Here we may assume that $\beta_{1} \leq \beta_{2}$. If $\beta_{1}=2$, then $D^{\#}=D_{2}^{\#}$ and so $\bar{P}_{2}(V-D)=\bar{P}_{2}\left(V-D_{2}\right)>0$ by Lemma 2.11 . Hence we may assume further that $\beta_{1} \geq 3$. We infer from Lemma 2.4 that

$$
0 \leq\left(D^{\#}+K_{V}\right)^{2}=\left(K_{V} \cdot D^{\#}+K_{V}\right)=\left(K_{V}^{2}\right)-8+\sum_{i=1}^{2}\left(\beta_{i}+\frac{4}{\beta_{i}}\right) .
$$

By using the Riemann-Roch theorem, $h^{2}\left(V, D+2 K_{V}\right)=h^{0}\left(V,-\left(D+K_{V}\right)\right)=0$ (cf. Proof of Lemma 2.11) and the above inequality, we have

$$
\begin{aligned}
h^{0}\left(V, D+2 K_{V}\right) & =h^{0}\left(V, D+2 K_{V}\right)+h^{2}\left(V, D+2 K_{V}\right) \\
& \geq \frac{1}{2}\left(D+2 K_{V} \cdot D+K_{V}\right)+1 \\
& =-5+\beta_{1}+\beta_{2}+\left(K_{V}^{2}\right) \\
& \geq 3-\left(\frac{4}{\beta_{1}}+\frac{4}{\beta_{2}}\right) \geq \frac{1}{3}>0 .
\end{aligned}
$$

Hence, $P_{2}[B]=\bar{P}_{2}(V-D)>0$.

Theorem 1.1 is thus proved. 


\section{Structure of $(X, B)$ with $\kappa[B]=0$ or 1}

In this section, we prove the following result.

THEOREM 4.1. Let $(X, B)$ be a pair of a smooth projective rational surface and a reduced curve $B$ on $X$ and let $(V, D)$ be an almost minimal model of $(X, B)$. Assume that $B$ consists of two rational curves and $\kappa[B]=0$ or 1 . Then we have:

(1) There exists an elliptic fibration $h: V \rightarrow \mathbf{P}^{1}$ such that every component of $D$ is contained in a fiber of $h$.

(2) If $D$ is irreducible, then $\left(D^{2}\right)=-4$.

(3) Assume that $D$ consists of two curves $D_{1}$ and $D_{2}$. If $\left(D_{1} \cdot D_{2}\right)=0$, then $\left\{\left(D_{1}^{2}\right),\left(D_{2}^{2}\right)\right\}=\{-2,-4\}$ or $\{-4,-4\}$. If $\left(D_{1} \cdot D_{2}\right)>0$, then $\left(D_{1} \cdot D_{2}\right)=1$ and $\left\{\left(D_{1}^{2}\right),\left(D_{2}^{2}\right)\right\}=\{-3,-3\}$ or $\{-2,-5\}$. Moreover, if $\left\{\left(D_{1}^{2}\right),\left(D_{2}^{2}\right)\right\}=\{-2,-5\}$ (in this case, $\left.\left(D_{1} \cdot D_{2}\right)>0\right)$, then $\kappa[B]=1$.

In fact, we have more precise results as for the pairs $(V, D)$. See Lemmas 4.2, 4.3, 4.4, 4.7 and 4.9 for more details.

In what follows, we shall prove Theorem 4.1. Let the notation and assumptions be the same as in Theorem 4.1. We note that $\#(D)=1$ or 2 because $\#(B)=2$ and $\kappa[B] \geq 0$. By the method of construction of an almost minimal model of an SNC-pair, we know that $\bar{p}_{g}(V-D)=0$.

If $D$ is irreducible, then $\left(D^{2}\right) \leq-2$ by Lemma 2.10. So, in this case, the assertions of Theorem 4.1 follow from Lemma 4.2 below.

LEMMA 4.2. With the same notation as above, assume that $\#(D)=1$. Then we have:

(1) If $\kappa[B]=0$, then $\left(D^{2}\right)=-4$ and $D+2 K_{V} \sim 0$. Furthermore, if $E$ is any $(-1)$ curve, then the linear system $|D+2 E|$ is an irreducible pencil of elliptic curves. We have a birational morphism $f: V \rightarrow \mathbf{P}^{2}$ such that $f(D)$ is a sextic with ten double points (possibly including infinitely near points).

(2) If $\kappa[B]=1$, then $\left(D^{2}\right)=-4$ and $\left|D+3 K_{V}\right| \neq \emptyset$. There exists a unique $(-1)$ curve $E_{0}$ such that $\left(E_{0} \cdot D\right)=2$. Furthermore, the linear system $\left|D+2 E_{0}\right|$ is an irreducible pencil of elliptic curves. There also exists a birational morphism $f: V \rightarrow \mathbf{P}^{2}$ such that $f(D)$ is a curve of degree $3 m, m \geq 3$ with nine $m$-tuple points and one double point (possibly including infinitely near points).

Proof. Note that $\kappa[B]=\bar{\kappa}(V-D)$. So the assertions (1) and (2) follow from [8, Theorem 3.3] (see also [2], [13]).

In the subsequent arguments, we assume further that $D$ is reducible. Let $D=D_{1}+D_{2}$ be the decomposition of $D$ into irreducible components and put $\beta_{i}:=-\left(D_{i}^{2}\right)$ for $i=1,2$. We may assume that $\beta_{1} \leq \beta_{2}$. 
Suppose that $\left(D_{1} \cdot D_{2}\right)>0$ (i.e., $D$ is connected). Then $\left(D_{1} \cdot D_{2}\right)=1$ and $D$ is an admissible rational rod by Lemma 2.10 and $\bar{p}_{g}(V-D)=0$. So, by the results of [8], we obtain the following results (Lemmas 4.3 and 4.4).

Lemma 4.3. Assume that $D$ is reducible and connected and that $\kappa[B]=0$. Then, we have:

(1) $\beta_{1}=\beta_{2}=3$.

(2) $D+2 K_{V} \sim 0$. In particular, $\left(K_{V}^{2}\right)=-1$.

(3) There exists a (-1)-curve $E$ on $V$ such that the linear system $|D+2 E|$ defines an irreducible pencil of elliptic curves. Namely, $V$ has an elliptic fibration over $\mathbf{P}^{1}$ which contains $D$ in a fiber.

Proof. The assertions follow from [8, Theorems 2.3 and 4.1].

Lemma 4.4. Assume that $D$ is reducible and connected and that $\kappa[B]=1$. Then, we have:

(1) $\left(\beta_{1}, \beta_{2}\right)=(3,3)$ or $(2,5)$. In particular, $\left(K_{V}^{2}\right)=-1$.

(2) There exists an elliptic fibration $h: V \rightarrow \mathbf{P}^{1}$ such that $D$ is contained in a fiber $F_{0}$ of $h$. Moreover, $\left(F_{0}\right)_{\mathrm{red}}=D+E_{0}$, where $E_{0}$ is a $(-1)$-curve such that $\left(E_{0} \cdot D_{1}\right)=$ $\left(E_{0} \cdot D_{2}\right)=1$.

PROOF. The assertions follow from [8, Theorem 2.5].

From now on, we consider the case $\left(D_{1} \cdot D_{2}\right)=0$ (i.e., $D$ is not connected). Then $\beta_{i} \geq 2$ for $i=1,2$ and $D^{\#}=\left(\left(\beta_{1}-2\right) / \beta_{1}\right) D_{1}+\left(\left(\beta_{2}-2\right) / \beta_{2}\right) D_{2}$. Since $D^{\#}+K_{V}$ is nef and $\left(D^{\#}+K_{V}\right)^{2}=0$ by Lemma 2.4, we have

$$
0=\left(D^{\#}+K_{V}\right)^{2}=\left(K_{V} \cdot D^{\#}+K_{V}\right)=\left(K_{V}^{2}\right)+\sum_{i=1}^{2} \frac{\left(\beta_{i}-2\right)^{2}}{\beta_{i}} .
$$

So, we obtain the following six cases:

$\begin{array}{ll}\text { Case 1). } & \beta_{1}=2, \beta_{2}=4 . \\ \text { Case 2). } & \beta_{1}=\beta_{2}=4 . \\ \text { Case 3). } & \beta_{1}=3, \beta_{2}=6 . \\ \text { Case 4). } & \beta_{1}=5, \beta_{2}=20 . \\ \text { Case 5). } & \beta_{1}=6, \beta_{2}=12 . \\ \text { Case 6). } & \beta_{1}=\beta_{2}=8 .\end{array}$

We prove Lemmas 4.5 and 4.6 which are essentially proved in [4, pp. 372-374].

LEMMA 4.5. With the same notation and assumptions as above, the cases 4) 6) do not take place.

Proof. Suppose that $\left(\beta_{1}, \beta_{2}\right)=(5,20)$. Then $\left(K_{V}^{2}\right)=-18$. So $V$ is not relatively minimal. If $\bar{\kappa}(V-D)=0$, then $D^{\#}+K_{V} \equiv 0$ by Lemma 2.6. For any (-1)-curve $E$ on $V$, we have $0=\left(E \cdot D^{\#}+K_{V}\right)=-1+\frac{3}{5}\left(D_{1} \cdot E\right)+\frac{9}{10}\left(D_{2} \cdot E\right)$. This derives a contradiction 
because both $\left(D_{1} \cdot E\right)$ and $\left(D_{2} \cdot E\right)$ are non-negative integers. Assume that $\bar{\kappa}(V-D)=1$. Then, by Lemma 2.7, the linear system $\left|j\left(D^{\#}+K_{V}\right)\right|(j>>0)$ defines an elliptic fibration $h: V \rightarrow \mathbf{P}^{1}$ and $D_{1}$ and $D_{2}$ are contained in fibers of $h$. Since $\left(K_{V}^{2}\right)=-18<0, h$ is not relatively minimal. Let $E$ be a $(-1)$-curve contained in a fiber of $h$ (which exists). Then $\left(E \cdot D^{\#}+K_{V}\right)=0$. So, we can derive a contradiction by using the same argument as in the case $\bar{\kappa}(V-D)=0$. Thus, we know that Case 4) does not take place. Similarly to Case 4), we can derive contradictions also in the cases 5) and 6).

LEMMA 4.6. With the same notation and assumptions as above, assume further that the case 3$)$ takes place. Then $\bar{\kappa}(V-D)=1$.

Proof. Suppose that $\bar{\kappa}(V-D)=0$. Then $D^{\#}+K_{V} \equiv 0$ by Lemma 2.6. Since $\left(D_{1}^{2}\right)=-3>-4$, we have $\bar{\kappa}\left(V-D_{1}\right)=-\infty$ by [8, Theorem 3.3] (see also [4, Lemma 1]). If $\left(V, D_{1}\right)$ is almost minimal, then it follows from [8, Theorem $\left.3.3(1)\right]$ that $\left(V, D_{1}\right)=$ $\left(\Sigma_{\beta_{1}}, M_{\beta_{1}}\right)$, where $\Sigma_{\beta_{1}}$ is a Hirzebruch surface of degree $\beta_{1}$ and $M_{\beta_{1}}$ is the minimal section of $\Sigma_{\beta_{1}}$. However, this is a contradiction. So, $\left(V, D_{1}\right)$ is not almost minimal. Then there exists a $(-1)$-curve $E$ on $V$ such that $\left(E \cdot D_{1}\right) \leq 1$. Since

$$
0=\left(D^{\#}+K_{V} \cdot E\right)=\frac{1}{3}\left(D_{1} \cdot E\right)+\frac{2}{3}\left(D_{2} \cdot E\right)-1
$$

we know that $\left(E \cdot D_{1}\right)=\left(E \cdot D_{2}\right)=1$.

Let $g: V \rightarrow V_{1}$ be the contraction of $E$ and put $D_{i}^{\prime}=g\left(D_{i}\right), i=1,2$. Then $\left(D_{1}^{\prime}\right)^{2}=$ $-2,\left(D_{2}^{\prime}\right)^{2}=-5,\left(D_{1}^{\prime} \cdot D_{2}^{\prime}\right)=1$ and $\left(K_{V_{1}}^{2}\right)=-2$. Moreover, we can easily see that $\left(D^{\prime}\right)^{\#}=\frac{1}{3} D_{1}^{\prime}+\frac{2}{3} D_{2}^{\prime}$ and $\left(D^{\prime}\right)^{\#}+K_{V_{1}}=g_{*}\left(D^{\#}+K_{V}\right) \equiv 0$. Hence the pair $\left(V_{1}, D^{\prime}\right)$ is almost minimal and $\bar{\kappa}\left(V_{1}-D^{\prime}\right)=0$. This contradicts Lemma 4.3.

We consider the two cases $\bar{\kappa}(V-D)=0$ and $\bar{\kappa}(V-D)=1$ separately.

CASE 1: $\bar{\kappa}(V-D)=1$. Lemma 2.7 implies that there exists an elliptic fibration $h: V \rightarrow \mathbf{P}^{1}$ such that $D_{1}$ and $D_{2}$ are contained in fibers of $h$. Let $F_{i}(i=1,2)$ be the fiber of $h$ containing $D_{i}$.

SubCASE 1-1: $\left(\beta_{1}, \beta_{2}\right)=(2,4)$. In this subcase, $\left(K_{V}^{2}\right)=-1$. So there exists a $(-1)$-curve $E$ contained in a fiber of $h$. Since $\left(E \cdot D^{\#}+K_{V}\right)=0$ and $D^{\#}=\frac{1}{2} D_{2}$, we have $\left(E \cdot D_{2}\right)=2$. Hence we know that $F_{1} \neq F_{2}$ and $F_{2}=n\left(D_{2}+2 E\right)$ for some positive integer $n$.

SubCASE 1-2: $\beta_{1}=\beta_{2}=4$. In this subcase, $D^{\#}=\frac{1}{2} D$ and $\left(K_{V}^{2}\right)=-2$. So there exists a $(-1)$-curve $E$ contained in a fiber of $h$. Since $\left(E \cdot D^{\#}+K_{V}\right)=0$, either $\left(E \cdot D_{1}\right)=$ $\left(E \cdot D_{2}\right)=1$ or $(E \cdot D)=\left(E \cdot D_{i}\right)=2$ for $i=1$ or 2 .

Assume that $\left(E \cdot D_{1}\right)=\left(E \cdot D_{2}\right)=1$. Let $\mu: V \rightarrow V_{1}$ be the contraction of $E$ and put $D_{i}^{\prime}:=\mu\left(D_{i}\right)(i=1,2)$ and $D^{\prime}:=\mu_{*}(D)$. Then $\left(D^{\prime}\right)^{\#}=\frac{1}{2} D^{\prime}$ and $\left(D^{\prime}\right)^{\#}+K_{V_{1}}=$ 
$\mu_{*}\left(D^{\#}+K_{V}\right)$. So the pair $\left(V_{1}, D^{\prime}\right)$ is almost minimal and $\bar{\kappa}\left(V_{1}-D^{\prime}\right)=1$. Hence, the pair $\left(V_{1}, D^{\prime}\right)$ is the one in Lemma 4.4 .

Assume that $(E \cdot D)=\left(E \cdot D_{i}\right)=2, i=1$ or 2 . We may assume $i=1$. Then, $F_{1}=n\left(D_{1}+2 E\right)$ for some positive integer $n$ because $\left(D_{1}+2 E\right)^{2}=0$. Since $\left(K_{V}^{2}\right)=-2$, there exists another $(-1)$-curve, say $E^{\prime}$, contained in a fiber of $h$. Then we can easily see that $\left(E^{\prime} \cdot D\right)=\left(E^{\prime} \cdot D_{2}\right)=2$ and $F_{2}=n^{\prime}\left(D_{2}+2 E^{\prime}\right)$ for some positive integer $n^{\prime}$.

SubCASE 1-3: $\quad\left(\beta_{1}, \beta_{2}\right)=(3,6)$. In this subcase, $D^{\#}=\frac{1}{3} D_{1}+\frac{2}{3} D_{2}$ and $\left(K_{V}^{2}\right)=-3$. So there exists a $(-1)$-curve $E$ contained in a fiber of $h$. Since $\left(E \cdot D^{\#}+K_{V}\right)=0$, we know that $\left(\left(E \cdot D_{1}\right),\left(E \cdot D_{2}\right)\right)=(3,0)$ or $(1,1)$. If $\left(\left(E \cdot D_{1}\right),\left(E \cdot D_{2}\right)\right)=(3,0)$, then $\left(E+D_{1}\right)^{2}=$ $2>0$, which is a contradiction because $E, D_{1} \subset \operatorname{Supp}\left(F_{1}\right)$. Hence $\left(E \cdot D_{1}\right)=\left(E \cdot D_{2}\right)=1$. In particular, $F_{1}=F_{2}$.

Let $\mu: V \rightarrow V_{1}$ be the contraction of $E$ and put $D_{i}^{\prime}=\mu\left(D_{i}\right)(i=1,2)$ and $D^{\prime}=$ $D_{1}^{\prime}+D_{2}^{\prime}$. Then $D^{\prime \#}=\frac{1}{3} D_{1}^{\prime}+\frac{2}{3} D_{2}^{\prime}$ and $D^{\prime \#}+K_{V_{1}}=\mu_{*}\left(D^{\#}+K_{V}\right)$. So the pair $\left(V_{1}, D^{\prime}\right)$ is almost minimal and $\bar{\kappa}\left(V_{1}-D^{\prime}\right)=1$. Hence, the pair $\left(V_{1}, D^{\prime}\right)$ is the one in Lemma 4.4.

By the argument as in Case 1, we obtain the following result.

Lemma 4.7. Assume that $D=D_{1}+D_{2}$ is not connected and $\bar{\kappa}(V-D)=1$. Then $\left(\beta_{1}, \beta_{2}\right)=(2,4),(4,4)$ or $(3,6)$. Moreover, we have:

(1) If $\left(\beta_{1}, \beta_{2}\right)=(2,4)$, then the pair $\left(V, D_{2}\right)$ is the one in Lemma 4.2 (2) and the curve $D_{1}$ is contained in a fiber of the elliptic fibration $h: V \rightarrow \mathbf{P}^{1}$ defined by $\left|j\left(D^{\#}+K_{V}\right)\right|$ $(j>>0)$.

(2) If $\beta_{1}=\beta_{2}=4$, then $D$ is contained in one or two fibers of the elliptic fibration $h: V \rightarrow \mathbf{P}^{1}$ defined by $\left|j\left(D^{\#}+K_{V}\right)\right|(j>>0)$.

(3) If $\left(\beta_{1}, \beta_{2}\right)=(3,6)$, then there exists a $(-1)$-curve $E$ such that $\left(E \cdot D_{1}\right)=(E$. $\left.D_{2}\right)=1$. Moreover, if $\mu: V \rightarrow V_{1}$ be the contraction of $E$, then the pair $\left(V_{1}, \mu(D)\right)$ is the one in Lemma 4.4.

CASE 2: $\quad \bar{\kappa}(V-D)=0$. In this case, $D^{\#}+K_{V} \equiv 0$ by Lemma 2.6. By Lemmas 4.5 and $4.6,\left(\beta_{1}, \beta_{2}\right)=(2,4)$ or $(4,4)$.

SUBCASE 2-1: $\quad \beta_{1}=\beta_{2}=4$. Since $D^{\#}+K_{V}=\frac{1}{2} D+K_{V} \equiv 0$, we have $(D \cdot E)=2$ for any $(-1)$-curve $E$. We note that there exist no $(-m)$-curves $(m \geq 3)$ other than $D_{1}$ and $D_{2}$. By [4, Lemma 3], we obtain a birational morphism $f: V \rightarrow \mathbf{P}^{2}$. Then $f(D)$ is a curve of degree six. So we can find a $(-1)$-curve $E$ on $V$ such that $\left(E \cdot D_{1}\right)=\left(E \cdot D_{2}\right)=1$. Let $\mu: V \rightarrow V_{1}$ be the contraction of $E$ and put $D_{i}^{\prime}=\mu\left(D_{i}\right)(i=1,2)$ and $D^{\prime}=D_{1}^{\prime}+D_{2}^{\prime}$. Then $D^{\prime \#}=\frac{1}{2} D^{\prime}$ and $D^{\prime \#}+K_{V_{1}}=\mu_{*}\left(D^{\#}+K_{V}\right) \equiv 0$. Hence the pair $\left(V_{1}, D^{\prime}\right)$ is almost minimal and $\bar{\kappa}\left(V_{1}-D^{\prime}\right)=0$. The pair $\left(V_{1}, D^{\prime}\right)$ is the one in Lemma 4.3. In particular, there exists an elliptic fibration $h: V \rightarrow \mathbf{P}^{1}$ such that $D$ is contained in a fiber of $h$. 
SubCASE 2-2: $\quad\left(\beta_{1}, \beta_{2}\right)=(2,4)$. In this subcase, $D^{\#}=\frac{1}{2} D_{2}$ and $\left(K_{V}^{2}\right)=-1$. Since $D_{2}^{\#}+K_{V}=D^{\#}+K_{V} \equiv 0$, the pair $\left(V, D_{2}\right)$ is almost minimal and $\bar{\kappa}\left(V-D_{2}\right)=0$. So the pair $\left(V, D_{2}\right)$ is the one in Lemma $4.2(1)$. Since $D_{1}$ is a $(-2)$-curve disjoint from $D_{2}$, it follows from Lemma 4.8 below that there exists a $(-1)$-curve $E$ disjoint from $D_{1}$. By Lemma 4.2 (1), the linear system $\left|D_{2}+2 E\right|$ defines an elliptic fibration $h: V \rightarrow \mathbf{P}^{1}$ and $D_{1}$ is contained in a fiber of $h$.

The following lemma is proved in [10, Lemma 2.1]. For the reader's convenience, we reproduce the proof.

LEMMA 4.8. Let $V$ be a smooth projective rational surface and let $D$ be a smooth rational curve on $V$. Assume that $\left(D^{2}\right)=-4$ and $D+2 K_{V} \sim 0$. (Note that the pair $(V, D)$ is the one in Lemma $4.2(1))$. Let $F$ be a (-2)-curve on $V$. Then there exists a (-1)-curve $E$ such that $E \cap F=\emptyset$.

PRoOF. By virtue of [14, Theorem 1], there exists a birational morphism $\rho: V \rightarrow \Sigma_{r}$ (onto a Hirzebruch surface of degree $r$ ) such that $r \neq 1$ and one of the following three cases takes place:

(1) $\rho(F)$ is a point.

(2) $\rho(F)$ is a fiber of the fixed ruling on $\Sigma_{r}$.

(3) $\rho(F) \sim M_{r}+n \ell$ with $2 n=r-2$, where $M_{r}$ is the minimal section of $\Sigma_{r}$ and $\ell$ is a fiber of the fixed ruling on $\Sigma_{r}$.

In the cases (2) and (3), we note that $\rho(F)$ does not pass through any one of the fundamental points of $\rho$ (cf. [14, Theorem 1]).

CASEs (2) and (3). Since $\left(K_{V}^{2}\right)=-1$ and $\left(K_{\Sigma_{r}}^{2}\right)=8, \Sigma_{r}$ has an fundamental point, say $P$, of $\rho^{-1}$. Then $\rho^{-1}(P)$ contains a $(-1)$-curve $E$. Since $P \notin \rho(F), E \cap F=\emptyset$.

CASE (1). Since $D+2 K_{V} \sim 0$, we have $\rho_{*}(D)+2 K_{\Sigma_{r}} \sim 0$. So, $\rho^{\prime}\left(M_{r}\right) \neq D$. On the other hand, if $A$ is an irreducible curve on $V$ such that $A \neq D$, then $\left(A \cdot K_{V}\right) \leq 0$, whence $\left(A^{2}\right)=2 p_{a}(A)-2-\left(A \cdot K_{V}\right) \geq-2$. Hence, taking $A$ to be $\rho^{\prime}\left(M_{r}\right)$, we have $\left(\rho^{\prime}\left(M_{r}\right)^{2}\right) \geq-2$. So, $r \leq 2$ and if $r=2$ then there exist no fundamental points of $\rho$ on $M_{r}$. Therefore, we obtain a birational morphism $\tau: V \rightarrow \mathbf{P}^{2}$ such that $\tau(F)$ is a point or a line.

Suppose first that $B:=\tau(F)$ is a line. Since $\left(F^{2}\right)=-2$, there lie three fundamental points of $\tau^{-1}$ on $B$, which might be infinitely near points. Since $\left(K_{V}^{2}\right)=-1$, there exists a fundamental point which does not line on $B$. Hence, we can find a $(-1)$-curve $E$ such that $F \cap E=\emptyset$.

Suppose next that $\tau(F)$ is a point, say $P$. By the same argument as above, it is easy to show that there exists an $(-1)$-curve $E$ with $F \cap E=\emptyset$, except the case where $\tau^{-1}(P)=$ $E+F+F_{1}+\cdots+F_{8}$ consists of a $(-1)$-curve $E$ and nine (-2)-curves $F, F_{1}, \cdots, F_{8}$ such that $(E \cdot D)=2$ and $E+F+F_{1}+\cdots+F_{8}$ is a linear chain with $(E \cdot F)=\left(F \cdot F_{1}\right)=$ $\cdots=\left(F_{7} \cdot F_{8}\right)=1$. Lemma $4.2(2)$ implies that $|D+2 E|$ defines an elliptic fibration 
$\Phi:=\Phi_{|D+2 E|}: V \rightarrow \mathbf{P}^{1}$. Then $F_{1}+\cdots+F_{8}$ is contained in a fiber, say $G$, of $\Phi$. Since $\left(K_{V}^{2}\right)=-1, E$ is the unique $(-1)$-curve contained in the fibers of $\Phi$. Let $\ell$ be a line on $\mathbf{P}^{2}$ passing through $P$ and the next immediate infinitely near fundamental point of $\tau^{-1}$. Let $\rho_{1}: V_{1} \rightarrow \mathbf{P}^{2}$ be the blowing-up of $\mathbf{P}^{2}$ with center $P$, let $G_{1}:=\rho_{1}^{-1}(P)$ and let $\ell^{(1)}:=\rho_{1}^{\prime}(\ell)$. Then $\tau: V \rightarrow \mathbf{P}^{2}$ is factored as $V \stackrel{\tau_{1}}{\longrightarrow} V_{1} \stackrel{\rho_{1}}{\longrightarrow} \mathbf{P}^{2}$, and $P_{1}:=G_{1} \cap \ell^{(1)}$ is a fundamental point of $\tau_{1}: V \rightarrow V_{1}$. Let $\rho_{2}: V_{2} \rightarrow V_{1}$ be the blowing-up of $V_{1}$ with center $P_{1}$, let $G_{2}:=\rho_{2}^{-1}\left(P_{1}\right)$, let $\ell^{(2)}:=\rho_{2}^{\prime}\left(\ell^{(1)}\right)$ and let $P_{2}:=G_{2} \cap \ell^{(2)}$.

Suppose that $P_{2}$ is a fundamental point of $\tau_{2}:=\rho_{2}^{-1} \circ \tau_{1}: V \rightarrow V_{2}$. Then, let $\rho_{3}:$ $V_{3} \rightarrow V_{2}$ be the blowing-up of $V_{2}$ with center $P_{2}$, let $\ell^{(3)}:=\rho_{3}^{\prime}\left(\ell^{(2)}\right)$, let $G_{3}:=\rho_{3}^{-1}\left(P_{2}\right)$ and let $P_{3}:=G_{3} \cap \ell^{(3)}$. Then $P_{3}$ is not a fundamental point of $\tau_{3}:=\rho_{3}^{-1} \circ \tau_{2}: V \rightarrow V_{3}$, for, otherwise, we get an irreducible curve $A:=\tau_{3}^{\prime}\left(\ell^{(3)}\right)$ such that $\left(A^{2}\right) \leq-3$ and $A \neq D$. Hence we know that $\left(\tau^{\prime}(\ell)^{2}\right)=-2, \tau^{\prime}(\ell)$ is a component of $G$ and

$$
\left(\tau^{\prime}(\ell) \cdot \sum_{i=1}^{8} F_{i}\right)=\left(\tau^{\prime}(\ell) \cdot F_{6}\right)=1 .
$$

Since

$$
\left(F_{1}+2 F_{2}+3 F_{3}+4 F_{4}+5 F_{5}+6 F_{6}+4 F_{7}+2 F_{8}+3 \tau^{\prime}(\ell)\right)^{2}=0,
$$

we have

$$
G=n\left(F_{1}+2 F_{2}+3 F_{3}+4 F_{4}+5 F_{5}+6 F_{6}+4 F_{7}+2 F_{8}+3 \tau^{\prime}(\ell)\right)
$$

for some positive integer $n$. Namely, $G$ is a singular fiber of type II* (cf. [7]). Then $G$ cannot be a multiple fiber by [7]. So, $n=1$ and

$$
(G \cdot F)=\left(F_{1} \cdot F\right)=1 \neq 2=(D+2 E \cdot F) .
$$

This is a contradiction. Therefore, $P_{2}$ is not a fundamental point of $\tau_{2}$. Then $\tau_{2}^{\prime}\left(\ell^{(2)}\right)$ is a $(-1)$-curve disjoint from $F$.

By the argument as in Case 2, we obtain the following result.

Lemma 4.9. Assume that $D=D_{1}+D_{2}$ is not connected and $\bar{\kappa}(V-D)=0$. Then $\left(\beta_{1}, \beta_{2}\right)=(2,4)$ or $(4,4)$. Moreover, we have:

(1) If $\left(\beta_{1}, \beta_{2}\right)=(2,4)$, then there exists a $(-1)$-curve $E$ such that $(E \cdot D)=\left(E \cdot D_{2}\right)=$ 2. The linear system $\left|D_{2}+2 E\right|$ defines an elliptic fibration $h: V \rightarrow \mathbf{P}^{1}$ where $D_{1}$ and $D_{2}$ are contained in fibers of $h$.

(2) If $\left(\beta_{1}, \beta_{2}\right)=(4,4)$, then there exists an elliptic fibration $h: V \rightarrow \mathbf{P}^{1}$ such that $D$ is contained in a fiber of $h$.

The proof of Theorem 4.1 is thus completed. 


\section{The case $\#(B)=3$ or 4}

In this section, we prove the following result.

THEOREM 5.1. Let $B$ be a reduced curve on a smooth projective rational surface. Assume that $\#(B) \leq 4$. Then $\kappa[B] \geq 0$ if and only if $P_{6}[B]>0$.

Let the notation be the same as in $\S 3$. In order to prove Theorem 5.1, it suffices to show that $\bar{P}_{6}(V-D)>0$ provided $\bar{\kappa}(V-D) \geq 0$ and $n:=\#(D)=3$ or 4 . We may assume that $\bar{p}_{g}(V-D)=0$, that is, each irreducible component of $D$ is a (smooth) rational curve and the dual graph of each connected component of $D$ is a tree (cf. Lemma 2.8).

Let $D=\sum_{i=1}^{n} D_{i}$ be the decomposition of $D$ into irreducible components and put $\beta_{i}:=-\left(D_{i}^{2}\right)$ for $i=1, \cdots, n$.

CASE 1: $n=3$. We consider the following three subcases separately.

SubCASE 1-1. If $D$ is connected, then $D$ is an admissible rational rod by the assumption $\bar{p}_{g}(V-D)=0$ and Lemma 2.10. So we have $\bar{P}_{2}(V-D)>0$ by Lemma 2.11 .

SubCASE 1-2. Assume that $D$ has two connected components. Then we may assume further that $D_{1}$ is a connected component of $D$. By Lemma 2.10, $D_{1}$ and $D_{2}+D_{3}$ are admissible rational rods. So we have

$$
D^{\#}=\left(\frac{\beta_{1}-2}{\beta_{1}}\right) D_{1}+\left(1-\frac{\beta_{3}+1}{\beta_{2} \beta_{3}-1}\right) D_{2}+\left(1-\frac{\beta_{2}+1}{\beta_{2} \beta_{3}-1}\right) D_{3} .
$$

If one of $D_{1}$ and $D_{2}+D_{3}$ is a $(-2)$-rod, then $\bar{P}_{2}(V-D)>0$ by virtue of Lemma 2.11. So we may assume that $\beta_{1} \geq 3$ and $\left(\beta_{2}, \beta_{3}\right) \neq(2,2)$. By Lemma 2.3 , we have

$$
\begin{aligned}
0 & \leq\left(D^{\#}+K_{V}\right)^{2}=\left(K_{V} \cdot D^{\#}+K_{V}\right) \\
& =\left(K_{V}^{2}\right)-10+\sum_{i=1}^{3} \beta_{i}+\frac{4}{\beta_{1}}+\frac{\beta_{2}+\beta_{3}+2}{\beta_{2} \beta_{3}-1} .
\end{aligned}
$$

Since $\beta_{1} \geq 3$ and $\left(\beta_{2}, \beta_{3}\right) \neq(2,2)$, we have

$$
\frac{4}{\beta_{1}}+\frac{\beta_{2}+\beta_{3}+2}{\beta_{2} \beta_{3}-1} \leq \frac{4}{3}+\frac{7}{5}=\frac{41}{15} .
$$

So, by using the Riemann-Roch theorem and $h^{2}\left(V, D+2 K_{V}\right)=h^{0}\left(V,-\left(D+K_{V}\right)\right)=0$ (cf. Proof of Lemma 2.11), we know that

$$
\begin{aligned}
h^{0}\left(V, D+2 K_{V}\right) & =h^{0}\left(V, D+2 K_{V}\right)+h^{2}\left(V, D+2 K_{V}\right) \\
& \geq \frac{1}{2}\left(D+2 K_{V} \cdot D+K_{V}\right)+1 \\
& =-7+\sum_{i=1}^{3} \beta_{i}+\left(K_{V}^{2}\right)
\end{aligned}
$$




$$
\begin{aligned}
& \geq 3-\frac{4}{\beta_{1}}-\frac{\beta_{2}+\beta_{3}+2}{\beta_{2} \beta_{3}-1} \\
& \geq 3-\frac{41}{15}>0 .
\end{aligned}
$$

Hence, $\bar{P}_{2}(V-D)>0$.

SubCASE 1-3. Assume that $D$ has three connected components. Then, by virtue of Theorem 1.1, we may assume that $\beta_{i} \geq 3$ for $i=1,2,3$. So,

$$
D^{\#}=\sum_{i=1}^{3}\left(\frac{\beta_{i}-2}{\beta_{i}}\right) D_{i} .
$$

By Lemma 2.3, we have

$$
0 \leq\left(D^{\#}+K_{V}\right)^{2}=\left(K_{V}^{2}\right)-12+\sum_{i=1}^{3}\left(\beta_{i}+\frac{4}{\beta_{i}}\right) .
$$

By using the Riemann-Roch theorem and $h^{2}\left(V, D+2 K_{V}\right)=0$ (cf. Proof of Lemma 2.11), we have

$$
\begin{aligned}
h^{0}\left(V, D+2 K_{V}\right) & =h^{0}\left(V, D+2 K_{V}\right)+h^{2}\left(V, D+2 K_{V}\right) \\
& \geq \frac{1}{2}\left(D+2 K_{V} \cdot D+K_{V}\right)+1 \\
& =-8+\sum_{i=1}^{3} \beta_{i}+\left(K_{V}^{2}\right) .
\end{aligned}
$$

Hence,

$$
h^{0}\left(V, D+2 K_{V}\right) \geq 4-4 \sum_{i=1}^{3} \frac{1}{\beta_{i}} .
$$

Suppose that $\bar{P}_{2}(V-D)=0$. Since $\beta_{i} \geq 3$ for $i=1,2,3$, it follows from the inequality as above that $\beta_{1}=\beta_{2}=\beta_{3}=3$. Moreover, $\left(D^{\#}+K_{V}\right)^{2}=0$ and $\left(K_{V}^{2}\right)=-1$. By Lemma $2.3, \bar{\kappa}(V-D)=0$ or 1 . Since $D+3 K_{V}=3\left(D^{\#}+K_{V}\right)$ is nef and $V$ is a rational surface, we have $h^{2}\left(V, D+3 K_{V}\right)=0$. So, by using the Riemann-Roch theorem, we have

$$
\begin{aligned}
h^{0}\left(V, D+3 K_{V}\right) & =h^{0}\left(V, D+3 K_{V}\right)+h^{2}\left(V, D+3 K_{V}\right) \\
& \geq \frac{1}{2}\left(D+3 K_{V} \cdot D+2 K_{V}\right)+1=1 .
\end{aligned}
$$

Hence, $\bar{P}_{3}(V-D)>0$.

Therefore, in this case, we know that $\bar{P}_{i}(V-D)>0$, where $i=2$ or 3. In particular, $\bar{P}_{6}(V-D)>0$. 
CASE 2. $n=4$. In this case, we may assume that the dual graph of $D$ is one of the following:

(i)

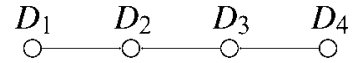

(iii) $\begin{array}{cccc}D_{1} & D_{2} & D_{3} & D_{4} \\ 0 & 0 & 0 & \bigcirc\end{array}$

(v) $\begin{array}{cccc}D_{1} & D_{2} & D_{3} & D_{4} \\ 0 & \bigcirc & 0 & 0\end{array}$

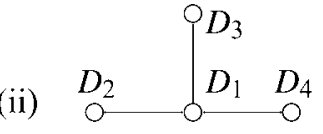

(iv) $\begin{array}{cccc}D_{1} & D_{2} & D_{3} & D_{4} \\ 0 & 0^{\circ} & 0 & 0\end{array}$

(vi) $\begin{array}{cccc}D_{1} & D_{2} & D_{3} & D_{4} \\ 0 & 0 & 0 & 0\end{array}$

We consider the following six subcases separately.

SUBCASE 2-(i). In this subcase, by Lemma $2.10, D$ is an admissible rational rod. Hence $\bar{P}_{2}(V-D)>0$ by Lemma 2.11 .

SubCASE 2-(ii). If $D$ is an admissible rational fork, then $\bar{P}_{2}(V-D)>0$ by Lemma 2.11. Assume that $D$ is not an admissible rational fork. By Lemma 2.10, $\beta_{i} \geq 2(i=2,3,4)$. So we have

$$
D^{\#}=D_{1}+\sum_{i=2}^{4}\left(\frac{\beta_{i}-1}{\beta_{i}}\right) D_{i}
$$

It follows from Lemma 2.3 that

$$
\begin{aligned}
0 & \leq\left(D^{\#}+K_{V}\right)^{2}=\left(D_{1} \cdot D^{\#}+K_{V}\right)+\left(K_{V} \cdot D^{\#}+K_{V}\right) \\
& =\left(K_{V}^{2}\right)-10+\sum_{i=1}^{4} \beta_{i}+\sum_{j=2}^{4} \frac{1}{\beta_{j}} .
\end{aligned}
$$

Since $\sum_{j=2}^{4} 1 / \beta_{i} \leq 3 / 2$, we know that $\bar{P}_{2}(V-D)>0$ by using a similar argument to Subcase 1-2.

SUBCASE 2-(iii). In this subcase, we may assume that both $D_{1}+D_{2}+D_{3}$ and $D_{4}$ are admissible rational rods, $\beta_{4} \geq 3$ and $\left(\beta_{1}, \beta_{2}, \beta_{3}\right) \neq(2,2,2)$. Then

$$
\begin{aligned}
D^{\#}= & \left(1-\frac{\beta_{2} \beta_{3}}{\beta_{1} \beta_{2} \beta_{3}-\left(\beta_{1}+\beta_{3}\right)}\right) D_{1}+\left(1-\frac{\beta_{1}+\beta_{3}}{\beta_{1} \beta_{2} \beta_{3}-\left(\beta_{1}+\beta_{3}\right)}\right) D_{2} \\
& +\left(1-\frac{\beta_{1} \beta_{2}}{\beta_{1} \beta_{2} \beta_{3}-\left(\beta_{1}+\beta_{3}\right)}\right) D_{3}+\left(\frac{\beta_{4}-2}{\beta_{4}}\right) D_{4} .
\end{aligned}
$$

It follows from Lemma 2.3 that

$$
0 \leq\left(D^{\#}+K_{V}\right)^{2}
$$




$$
=\left(K_{V}^{2}\right)-12+\sum_{i=1}^{4} \beta_{i}+\frac{\beta_{1} \beta_{2}+\beta_{2} \beta_{3}}{\beta_{1} \beta_{2} \beta_{3}-\left(\beta_{1}+\beta_{3}\right)}+\frac{4}{\beta_{4}} .
$$

Since $\beta_{4} \geq 3$ and $\left(\beta_{1}, \beta_{2}, \beta_{3}\right) \neq(2,2,2)$, we have $4 / \beta_{4} \leq 4 / 3$ and

$$
\frac{\beta_{1} \beta_{2}+\beta_{2} \beta_{3}}{\beta_{1} \beta_{2} \beta_{3}-\left(\beta_{1}+\beta_{3}\right)} \leq \frac{3}{2} \text {. }
$$

Hence we know that $\bar{P}_{2}(V-D)>0$ by using a similar argument to Subcase 1-2.

SUBCASE 2-(iv). In this subcase, we may assume that both $D_{1}+D_{2}$ and $D_{3}+D_{4}$ are admissible rational rods and $\left(\beta_{i}, \beta_{i+1}\right) \neq(2,2)$ for $i=1,3$. Then

$$
\begin{aligned}
D^{\#}= & \left(1-\frac{\beta_{2}+1}{\beta_{1} \beta_{2}-1}\right) D_{1}+\left(1-\frac{\beta_{1}+1}{\beta_{1} \beta_{2}-1}\right) D_{2} \\
& +\left(1-\frac{\beta_{4}+1}{\beta_{3} \beta_{4}-1}\right) D_{3}+\left(1-\frac{\beta_{3}+1}{\beta_{3} \beta_{4}-1}\right) D_{4} .
\end{aligned}
$$

It follows from Lemma 2.3 that

$$
\begin{aligned}
0 & \leq\left(D^{\#}+K_{V}\right)^{2} \\
& =\left(K_{V}^{2}\right)-12+\sum_{i=1}^{4} \beta_{i}+\frac{\beta_{1}+\beta_{2}}{\beta_{1} \beta_{2}-1}+\frac{\beta_{3}+\beta_{4}}{\beta_{3} \beta_{4}-1} .
\end{aligned}
$$

Since

$$
\frac{\beta_{i}+\beta_{i+1}}{\beta_{i} \beta_{i+1}-1} \leq \frac{7}{5}
$$

for $i=1,3$, we know that $\bar{P}_{2}(V-D)>0$ by using a similar argument to Subcase 1-2.

SUBCASE 2-(v). In this subcase, we may assume that $\beta_{1}, \beta_{2} \geq 3,2 \leq \beta_{3} \leq \beta_{4}$ and $\left(\beta_{3}, \beta_{4}\right) \neq(2,2)$. Then

$$
D^{\#}=\sum_{i=1}^{2}\left(\frac{\beta_{i}-2}{\beta_{i}}\right) D_{i}+\left(1-\frac{\beta_{4}+1}{\beta_{3} \beta_{4}-1}\right) D_{3}+\left(1-\frac{\beta_{3}+1}{\beta_{3} \beta_{4}-1}\right) D_{4} .
$$

It follows from Lemma 2.3 that

$$
\begin{aligned}
0 & \leq\left(D^{\#}+K_{V}\right)^{2} \\
& =\left(K_{V}^{2}\right)-14+\sum_{i=1}^{4} \beta_{i}+\frac{4}{\beta_{1}}+\frac{4}{\beta_{2}}+\frac{\beta_{3}+\beta_{4}+2}{\beta_{3} \beta_{4}-1} .
\end{aligned}
$$

By using the Riemann-Roch theorem and $h^{2}\left(V, D+2 K_{V}\right)=0$ (cf. Proof of Lemma 2.11), we have

$$
h^{0}\left(V, D+2 K_{V}\right)=h^{0}\left(V, D+2 K_{V}\right)+h^{2}\left(V, D+2 K_{V}\right)
$$




$$
\begin{aligned}
& \geq \frac{1}{2}\left(D+2 K_{V} \cdot D+K_{V}\right)+1 \\
& =\left(K_{V}^{2}\right)-10+\sum_{i=1}^{4} \beta_{i} \\
& \geq 4-\left(\frac{4}{\beta_{1}}+\frac{4}{\beta_{2}}+\frac{\beta_{3}+\beta_{4}+2}{\beta_{3} \beta_{4}-1}\right) .
\end{aligned}
$$

Hence, if $\bar{P}_{2}(V-D)=0$, then $\beta_{1}=\beta_{2}=\beta_{4}=3$ and $\beta_{3}=2$. Furthermore, we know that $\left(K_{V}^{2}\right)=-1$ and $\left(D^{\#}+K_{V}\right)^{2}=1 / 15$. In particular, $\bar{\kappa}(V-D)=2$ by Lemma 2.3.

We prove that $\bar{P}_{6}(V-D)>0$ in this subcase. Put $H=2 D_{1}+2 D_{2}+2 D_{3}+3 D_{4}+6 K_{V}$. Then $H$ is pseudo effective (that is, $(H \cdot A) \geq 0$ for any ample divisor $A$ ) because $H \geq$ $6\left(D^{\#}+K_{V}\right)$ and $D^{\#}+K_{V}$ is nef. Moreover, $h^{2}(V, H)=h^{0}\left(V, K_{V}-H\right)=0$ because $V$ is a rational surface. By using the Riemann-Roch theorem, we know that $h^{0}(V, H)>0$. Hence $\bar{P}_{6}(V-D)>0$.

SUBCASE 2-(vi). In this subcase, we may assume that $\beta_{i} \geq 3$ for $i=1, \cdots, 4$. Then $D^{\#}=\sum_{i=1}^{4}\left(\left(\beta_{i}-2\right) / \beta_{i}\right) D_{i}$. By using a similar argument to Subcase 2 -(v), we know that if $\bar{P}_{2}(V-D)=0$ then $\left(\beta_{1}, \beta_{2}, \beta_{3}, \beta_{4}\right)=(3,3,3,3)$ or $(3,3,3,4)$, where we assume that $\beta_{1} \leq \beta_{2} \leq \beta_{3} \leq \beta_{4}$

Assume that $\left(\beta_{1}, \beta_{2}, \beta_{3}, \beta_{4}\right)=(3,3,3,3)$. Then we know that $D^{\#}=\frac{1}{3} D,\left(K_{V}^{2}\right)=-1$ and $\bar{\kappa}(V-D)=2$. Moreover, $h^{2}\left(V, D+3 K_{V}\right)=0$ because $V$ is a rational surface and $D+3 K_{V}=3\left(D^{\#}+K_{V}\right)$ is nef and big. By using the Riemann-Roch Theorem, we know that $h^{0}\left(V, D+3 K_{V}\right)>0$. Hence, $\bar{P}_{3}(V-D)>0$.

Assume that $\left(\beta_{1}, \beta_{2}, \beta_{3}, \beta_{4}\right)=(3,3,3,4)$. Then, $D^{\#}=\frac{1}{3}\left(D_{1}+D_{2}+D_{3}\right)+\frac{1}{2} D_{4}$, $\left(K_{V}^{2}\right)=-2$ and $\left(D^{\#}+K_{V}\right)^{2}=0$. Hence $\bar{\kappa}(V-D)=0$ or 1 by Lemma 2.3. Since $D^{\#}+K_{V}$ is nef and $V$ is a rational surface, $h^{2}\left(V, 6\left(D^{\#}+K_{V}\right)\right)=h^{2}\left(V, 2\left(D_{1}+D_{2}+D_{3}\right)+3 D_{4}+\right.$ $\left.6 K_{V}\right)=0$. By using the Riemann-Roch theorem, we know that $h^{0}\left(V, 6\left(D^{\#}+K_{V}\right)\right)>0$. Hence, $\bar{P}_{6}(V-D)>0$.

Thus, in this case, we know that $\bar{P}_{6}(V-D)>0$.

The proof of Theorem 5.1 is thus completed.

\section{Remarks on the case $\#(B) \geq 5$}

Let $(X, B)$ be a pair of a smooth projective rational surface $X$ and a reduced curve $B$ on $X$ and let $(V, D)$ be an almost minimal model of $(X, B)$. By the results in [15], we obtain the following results.

Proposition 6.1. If $\kappa[B]=0$, then $P_{i}[B]=1$ for some $1 \leq i \leq 66$.

Proof. The assertion follows from [15, Theorem 2.1]. 
Proposition 6.2. Assume that $\left\lfloor D^{\#}\right\rfloor \neq 0$, where $\left\lfloor D^{\#}\right\rfloor$ denotes the integral part of $D^{\#}$, and $\kappa[B]=2$. Then $P_{12}[B]>0$.

Proof. The assertion follows from [15, Proposition 3.2].

Proposition 6.3. Assume that Supp $(D)$ is connected. Then $\kappa[B] \geq 0$ if and only if $P_{12}[B]>0$.

PROOF. The assertion follows from [15, Theorem 3.3].

The authors conjecture that, for any integer $n>0$, there exists an integer $N=N(n)$ such that $P_{N}[B]>0$ for any pair $(X, B)$ of a smooth projective rational surface $X$ and a reduced curve $B$ on $X$ with $\#(B)=n$ and $\kappa[B] \geq 0$.

\section{References}

[ 1 ] S. IITAKA, Algebraic Geometry, Graduate Texts in Math. 76, Springer (1981).

[2 ] S. IITAKA, On irreducible plane curves, Saitama Math. J. 1 (1983), 47-63.

[ 3 ] S. Iitaka, On a characterization of two lines on a projective plane, Proc. Algebraic Geometry, Lecture Notes in Math. 1016, Springer (1983) 432-448.

[ 4 ] S. IITAKA, Classification of reducible plane curves, Tokyo J. Math. 11 (1988), 363-379.

[ 5 ] S. IITAKA, Birational geometry of plane curves, Tokyo J. Math. 22 (1999), 289-321.

[ 6 ] Y. Kawamata, On the classification of non-complete algebraic surfaces, Proc. Copenhagen Summer meeting in Algebraic Geometry, Lecture Notes in Math. 732, Springer (1978), 215-232.

[ 7 ] K. KodAiRA, On compact analytic surfaces, II, Ann. of Math. (2) 77 (1963), 563-626.

[ 8 ] H. Kojima, Almost minimal embeddings of quotient singular points into rational surfaces, J. Math. Kyoto Univ. 38 (1998), 77-99.

[ 9 ] O. MatsudA, On numerical types of algebraic curves on rational surfaces, Tokyo J. Math. 24 (2001), 359367.

[10] R. Miranda, M. Miyanishi and M. Pavaman Murthy, Rational sextics with ten double points, unpublished manuscript.

[11] M. MiYanishi, Non-complete algebraic surfaces, Lecture Notes in Math. 857, Springer (1981).

[12] M. MiYanishi, Open algebraic surfaces, CRM Monograph Series 12, Amer. Math. Soc. (2000).

[13] N. Mohan Kumar and M. Pavaman Murthy, Curves with negative self intersection on rational surfaces, J. Math. Kyoto Univ. 22 (1983), 767-777.

[14] M. NAGATA, On rational surfaces, I, Mem. Coll. Sci. Univ. Kyoto 32 (1960), 351-370.

[15] S. TsunODA, Structure of open algebraic surfaces, I, J. Math. Kyoto Univ. 23 (1983), 95-125.

Present Addresses:

Hideo KOJIMA

Department of Mathematics, Faculty of Engineering, Niigata University,

NIIGATA, 950-2181 JAPAN.

e-mail: kojima@ie.niigata-u.ac.jp

TAKESHI TAKAHASHI

Division of General Education, Nagaoka National College of Technology, NishiKATAKAI, NAGAOKA, NiIGATA, 940-8532 JAPAN.

e-mail: takeshi@nagaoka-ct.ac.jp 